\title{
On sovereign default with time-varying interest rates ${ }^{\star}$
}

\author{
Gaetano Bloise $^{\mathrm{a}}$, Yiannis Vailakis $\mathrm{s}^{\mathrm{b}, *}$ \\ ${ }^{a}$ Yeshiva University, New York, United States and University of Rome III, Rome, Italy \\ ${ }^{b}$ Adam Smith Business School, University of Glasgow, United Kingdom
}

\begin{abstract}
We extend and refine Aguiar and Amador [3]'s contraction approach to Eaton and Gersovitz [14]'s sovereign debt model. In particular, we encompass time-varying interest rates and growth. We show that, when long-term interest rates exceed growth, equilibrium is unique and can be computed via contraction mapping. The method unifies separate branches of literature, showing that the contraction property is the reflection of previous arbitrage arguments based on replication, inspired by Bulow and Rogoff [13].
\end{abstract}

Keywords: Sovereign default, Time-varying interest rates, Uniqueness of equilibrium, Contraction mapping.

JEL Classifications: F34, F41.

\section{Introduction}

In this paper we provide a characterization of the canonical Eaton and Gersovitz [14]'s model of sovereign debt under time-varying interest rates and growth.

${ }^{\star}$ We thank the editor and two anonymous reviewers for their helpful comments. We are grateful to Mark Aguiar and Manuel Amador for discussions on the contraction approach to sovereign debt models. Gaetano Bloise acknowledges the financial support of the Italian Ministry of Education (PRIN 2015). Yiannis Vailakis acknowledges the financial support of an ERC starting grant (FP7, Ideas specific program, Project 240983 DCFM) and of two ANR research grants (Projects Novo Tempus and FIRE); also the hospitality of the Australian National University.

*Corresponding author

Email addresses: bloise@yu.edu (Gaetano Bloise), yiannis.vailakis@glasgow.ac.uk (Yiannis Vailakis) 
In particular, as in Aguiar and Amador [3], we show that equilibrium obtains as the unique fixed point of a contraction mapping, provided that interest rates are high relative to growth. The contraction property is the reflection of the more traditional arbitrage argument, originally inspired by Bulow and Rogoff [13] and recently exploited by Auclert and Rognlie [8] and Bloise et al. [10]. The contraction method immediately yields existence and uniqueness, unifying distinct strands of literature on Eaton and Gersovitz [14]'s model.

Aguiar and Amador [3] study a dual representation of equilibrium under a constant positive interest rate. In particular, as in Aguiar et al. [4], they consider a sort of minimum expenditure program, in which the amount of outstanding debt is minimized subject to ensuring a given level of utility to the sovereign. This dual transformation of the program allows them to show that equilibrium is determined as a fixed point of a contraction mapping, so establishing uniqueness by means of conventional recursive techniques. To gain a suitable space for the application of the contraction mapping principle, they impose an exogenous upper bound on consumption, a restriction that cannot account for economic growth, as it is instead the case in the quantitative literature (see, among others, Aguiar and Gopinath [5] and Arellano [7]).

We extend Aguiar and Amador [3]'s contraction mapping approach to a more general environment with time-varying interest rates and growth. However, differently from them, we work with the more natural primal representation of equilibrium and instead consider an adjusted (extended) metric on a suitable space of candidate equilibrium value functions. This seems preferable because the contraction property obtains straightaway. It emerges as the counterpart of the pure arbitrage argument originally used by Bulow and Rogoff [13]: any optimal plan with high borrowing can be replicated with lower level of debt along with an additional saving policy yielding a positive return.

Our general treatment identifies the critical assumption driving equilibrium uniqueness, so implicitly suggesting a channel towards multiplicity. The contraction property requires that long-term interest rates exceed economic growth, 
and it might fail otherwise. Thus, equilibrium uniqueness heavily relies on the widespread hypothesis of a constant positive interest rate. ${ }^{1}$ By contrast, old and recent empirical studies (Abel et al. [1], Blanchard [9] and Jordà et al. [16]) document that a low safe rate of interest, relative to growth, is more the historical norm than the exception for developed countries. A time-varying interest rate, recurrently falling below economic growth, reproduces these empirical patterns without necessarily permitting Ponzi games. Under these alternative conditions, sovereign debt markets are prone to self-fulfilling crises (multiple equilibria), as argued by Bloise and Vailakis [12].

Aguiar and Amador [3]'s contraction method, and the refinement we provide in this paper, might have other applications in macroeconomics. We indeed notice a rather enlightening feature of the approach: contrary to conventional applications, utility discounting plays no direct role in establishing the contraction property, which only relies on high interest rates relative to growth. Hence, the technique might also succeed in other frameworks in which discounting fails because of time non-additive or non-expected utility. Contraction obtains, irrespectively of discounting, provided that suitable lower and upper bounds exist.

Independently of the contraction approach, we enlarge the set of known conditions for a unique equilibrium in Eaton and Gersovitz [14]. Indeed, we only assume that the interest rate dominates growth in the long term rather than at all contingencies. Therefore, in the short term, and even for prolonged phases, interest rates might remain negative net of growth. To encompass these situations the analysis requires a major innovation, though the logic still rests upon a replication strategy firmly established in the literature. Auclert and Rognlie [8], for

\footnotetext{
${ }^{1}$ It is worth noticing that, as Aguiar and Amador [3], we assume exogenous values of default. Uniqueness might fail when the sovereign can re-gain market access after default, so that the value of default depends on the equilibrium value of borrowing. However, Auclert and Rognlie [8, Proposition 9] rule out any multiplicity involving distinct favorable and averse equilibria in which the favorable equilibrium offers uniformly better price than the averse equilibrium. Furthermore, Passadore and Xandri [17] show that multiplicity can arise when the sovereign is prevented from saving. This happens because the basic arbitrage argument enforcing uniqueness is precluded, as it would necessarily involve savings in some contingency.
} 
instance, adopt a 'mimicking at a distance' policy in which bond positions are increased by a constant amount corresponding to the maximum distance between distinct default thresholds. This simple arbitrage is unsuitable when the interest rate falls recurrently below growth. The replication policy becomes necessarily time-varying, involving plans of accumulation and decumulation of bonds over time depending on contingencies. The existence of these plans can hardly be established constructively and it is here that our method demonstrates its full potential.

We briefly relate the contribution in this paper to our previous work on debt sustainability. In all these papers we apply a dominant root method, developed in Bloise et al. [11], in order to capture tendencies of time-varying interest rates relative to growth. This machinery only serves to separate regimes for long-term interest rates without any direct substantive implication. Our objectives in the papers are rather different: in Bloise et al. [11] we argue that, at a competitive equilibrium under incomplete markets, debt can be sustained by the reputation for repayment alone; in Bloise and Vailakis [12] we show that low interest rates expose sovereign debt markets to an increased risk of self-fulfilling solvency crises; in this paper we extend and refine Aguiar and Amador [3]'s contraction approach to Eaton and Gersovitz [14]'s equilibrium. The papers are complement and, all together, they suggest that the conditions necessary for contraction are dramatically understated by the (not heuristic) assumption of a constant positive interest rate.

The paper is organized as follows. In section 2, we set up the model as in the established literature, but allowing for risk-averse creditors and temporal variation of interest rates. In section 3, we construct the primal equilibrium operator and introduce the adjusted metric. Finally, in section 4, we show that the equilibrium operator is a contraction in the adjusted metric. To capture long-term tendencies of interest rates, relative to growth, we adopt the dominant root method developed in Bloise et al. [11]. This permits to deal with time-varying interest rates at a level of tractability comparable with that of the more traditional constant interest rate. For completeness, in Appendix A, we verify robustness of our uniqueness 
theorem. In Appendix B, we provide a method to verify conditions on the dominant root by means of the spectral radius. In Appendix C, finally, we clarify that the contraction property does not require the interest rate to exceed (permanently) growth at all contingencies.

\section{Equilibrium}

We consider a general version of the sovereign debt model originally introduced by Eaton and Gersovitz [14]. ${ }^{2}$ The sovereign issues debt subject to limited commitment and might default when profitable. Bonds issued by the sovereign are traded in an international capital market populated by risk-averse investors. Comparing with previous studies, we allow for a time-varying safe interest rate and economic growth.

Uncertainty is represented by a Markov transition $\mu: S \rightarrow \Delta(S)$ on a discrete state space $S$. To avoid technical complications related to measurability issues, we assume that transitions are always on finite supports. Growth is permitted because the state space might be infinite. Furthermore, as we are silent on the nature of this process, we encompass any source of extrinsic uncertainty unrelated to fundamentals. Therefore, by showing uniqueness of equilibrium, we also establish the irrelevance of sunspots and market sentiments.

Assumption 1 (Markov uncertainty). Markov transition $\mu: S \rightarrow \Delta(S)$ is such that the support of $\mu(s)$ in $\Delta(S)$ is finite for every state $s$ in $S$, that is, the set $\left\{s^{\prime} \in S: \mu\left(s, s^{\prime}\right)>0\right\}$ is finite, where $\mu\left(s, s^{\prime}\right)$ is the probability of moving from state $s$ in $S$ to state $s^{\prime}$ in $S$.

International investors' valuation is described by a stochastic discount factor, or pricing kernel, $m$ in $\mathbb{R}^{S \times S}$ with $m\left(s, s^{\prime}\right)>0$ for all $\left(s, s^{\prime}\right)$ in $S \times S$. Timevariation of this process reflects both changes in creditors' exposure to sovereign default risk and the evolution of the safe rate of interest over time and across states.

\footnotetext{
${ }^{2}$ We refer to Aguiar and Amador [2] for an extensive survey of the literature.
} 
Absent default, the safe interest rate in state $s$ in $S$ is given by

$$
\frac{1}{1+r(s)}=\sum_{s^{\prime} \in S} \mu\left(s, s^{\prime}\right) m\left(s, s^{\prime}\right)
$$

where the sum is meaningful because of finite support (Assumption 1). The stochastic discount factor is primitive (i.e., exogenously given) and will serve to price default risk.

We impose an upper bound to the debt issued by the sovereign. This restriction is necessary because otherwise the sovereign will run a Ponzi scheme and default will never occur. This limit on outstanding debt might well be more permissive than the natural debt limit, the most pessimistic valuation of the borrower's endowment, because the sovereign might be defaulting at some future contingency and, so, solvency need not be ensured along all paths. In fact, as Aguiar and Amador [3], we shall allow for any finite bound not exceeding, whenever finite, some expansion of the most optimistic valuation of the sovereign's endowment.

Assumption 2 (Upper bound on debt). Debt can be issued only up to a finite positive limit $N$ in $\mathbb{R}^{S}$.

We now turn to the sovereign's optimal debt issuance. The sovereign begins with an inherited stock of bonds $b$ in $\mathbb{R}$, all reaching maturity in the current period. The sovereign's position is a claim if positive and a debt if negative. Nature then draws the current state $s$ in $S$. After observing this state, the government decides whether to repay its maturing debt or default. Upon default the sovereign secures a contingent reservation utility value $J^{D}(s)$ in $\mathbb{R}$. Conditional on debt repayment, instead, the government receives an endowment $y(s)$ in $\mathbb{R}^{++}$, auctions new bonds $b^{\prime}$ in $\mathbb{R}$, pays off the maturing debt, and consumes $c$ in $\mathbb{R}^{+}$. The sovereign maximizes expected discounted utility at a given discount factor $\delta$ in $(0,1)$. Preferences are restricted by canonical properties.

Assumption 3 (Utility). Utility function $u: \mathbb{R}^{+} \rightarrow \mathbb{R}$ is bounded, continuous and increasing. 
Assumption 4 (Reservation values). The reservation value upon default $J^{D}$ in $\mathbb{R}^{S}$ is bounded, that is,

$$
\sup _{s \in S}\left|J^{D}(s)\right| \text { is finite. }
$$

At equilibrium, the sovereign's borrowing plan is optimal subject to creditors' rational expectations about default. Default occurs whenever $J(s, b)<J^{D}(s)$, where $J(s, b)$ in $\mathbb{R}^{*}$ is the indirect utility given the initial bond holding $b$ in $\mathbb{R}$ when state $s$ in $S$ occurs. ${ }^{3}$ Otherwise, the optimal behavior of the sovereign is determined by the recursive program:

$$
J(s, b)=\sup _{\left(c, b^{\prime}\right) \in \mathbb{R}^{+} \times \mathbb{R}}(1-\delta) u(c)+\delta \sum_{s^{\prime} \in S} \mu\left(s, s^{\prime}\right) \max \left\{J\left(s^{\prime}, b^{\prime}\right), J^{D}\left(s^{\prime}\right)\right\}
$$

subject to

$$
Q\left(s, b^{\prime} \mid J\right)+c \leq y(s)+b
$$

and

$$
-N\left(s^{\prime}\right) \leq b^{\prime} \text { if } \mu\left(s, s^{\prime}\right)>0 .
$$

Restriction (2.1) is the budget constraint, whereas (2.2) prescribes that sovereign borrowing cannot exceed the given upper bound. Bonds issued by the sovereign $b^{\prime} \leq 0$ in $\mathbb{R}$ are priced under default risk, as we explain next.

Given bond holdings $b^{\prime}$ in $\mathbb{R}$, the default event in the next period is

$$
D\left(s, b^{\prime} \mid J\right)=\left\{s^{\prime} \in S: J\left(s^{\prime}, b^{\prime}\right)<J^{D}\left(s^{\prime}\right)\right\} .
$$

\footnotetext{
${ }^{3}$ Notice that, in order to cope with possibly empty feasible sets, we allow for extended real values $\mathbb{R}^{*}$, and we adhere to the convention that the value of the program is negative infinity $(J(s, b)=-\infty)$ when the feasible set is empty.
} 
The risk-premium on sovereign bonds is determined accordingly. That is,

$$
Q\left(s, b^{\prime} \mid J\right)=\left(\frac{1}{1+r(s)}\right) b^{\prime}-\sum_{s^{\prime} \in D\left(s, b^{\prime} \mid J\right)} \mu\left(s, s^{\prime}\right) m\left(s, s^{\prime}\right) \min \left\{b^{\prime}, 0\right\}
$$

Thus, creditors value bonds issued by the sovereign insofar as the sovereign remains solvent. This is the basic insight of Eaton and Gersovitz [14].

\section{An operator approach}

Inspired by Aguiar and Amador [3], we study equilibrium by means of an operator defined on a suitable space. We show that, when interest rates exceed growth in the long-term, the operator satisfies a contraction property and this ensures that equilibrium exists and is unique. Differently from Aguiar and Amador [3], we work on the primal sovereign maximization program. This allows us to establish a bridge between two separate strands of literature on equilibrium uniqueness in Eaton and Gersovitz [14]'s model.

Let $\mathcal{J}$ be the space of maps $J: S \times \mathbb{R} \rightarrow \mathbb{R}^{*}$ that are weakly increasing given $s$ in $S$ and bounded from above. The subspace $\mathcal{J}^{u} \subset \mathcal{J}$ consists of all maps $J: S \times \mathbb{R} \rightarrow \mathbb{R}^{*}$ that are also upper semicontinuous, that is, such that, for every $\epsilon$ in $\mathbb{R}$,

$$
\{b \in \mathbb{R}: J(s, b) \geq \epsilon\} \text { is closed. }
$$

The equilibrium operator $T: \mathcal{J} \rightarrow \mathcal{J}$ is defined by

$$
(T J)(s, b)=\sup _{\left(c, b^{\prime}\right) \in \mathbb{R}^{+} \times \mathbb{R}}(1-\delta) u(c)+\delta \sum_{s^{\prime} \in S} \mu\left(s, s^{\prime}\right) \max \left\{J\left(s^{\prime}, b^{\prime}\right), J^{D}\left(s^{\prime}\right)\right\}
$$

subject to constraints (2.1)-(2.2). We first establish that our construction is consistent.

Claim 1 (Monotonicity). Operator $T: \mathcal{J} \rightarrow \mathcal{J}$ is monotone and satisfies $\left(T \mathcal{J}^{u}\right) \subset$ $\mathcal{J}^{u}$. 
Proof. Monotonicity is obvious. It is also clear that $(T J): S \times \mathbb{R} \rightarrow \mathbb{R}^{*}$ is bounded from above and weakly increasing given state $s$ in $S$. So, we only have to prove the invariance condition for upper semicontinuous maps. Notice that the feasible correspondence is closed with compact values. Hence, by the Maximum Theorem (see Aliprantis and Border [6, Lemma 17.30]), $(T J)$ is upper semicontinuous given $s$ in $S$ and, so, an element of the restricted domain $\mathcal{J}^{u}$.

Let $\underline{J}$ in $\mathcal{J}^{u}$ be the value of the program under the additional restriction $b^{\prime} \geq 0$. Also, let $\bar{J}$ in $\mathcal{J}^{u}$ be the value of the program under the relaxed default-free pricing

$$
Q^{*}\left(s, b^{\prime}\right)=\left(\frac{1}{1+r(s)}\right) b^{\prime} .
$$

These values are well-defined by conventional dynamic programming arguments. ${ }^{4}$ We so immediately obtain suitable bounds for the operator.

Claim 2 (Bounds). Operator $T: \mathcal{J} \rightarrow \mathcal{J}$ satisfies

$$
\underline{J} \leq(T \underline{J}) \leq(T \bar{J}) \leq \bar{J} .
$$

Proof. The proof is immediate as the feasible set in the actual program is smaller than the one under the relaxed default-free pricing and larger than the one under the more restrictive no borrowing constraint.

We finally endow space $\mathcal{J}$ with an extended metric. To this purpose, let $f$ be a strictly positive element of $\mathbb{R}^{S}$. The distance $d_{f}\left(J^{\prime}, J^{\prime \prime}\right)$ in $\mathbb{R}^{*}$ is measured by the infimum over all $\epsilon$ in $\mathbb{R}^{+}$such that, for every $b$ in $\mathbb{R}$,

$$
J^{\prime}(s, b+\epsilon f(s)) \geq J^{\prime \prime}(s, b) \geq J^{\prime}(s, b-\epsilon f(s)),
$$

\footnotetext{
${ }^{4}$ Eaton and Gersovitz [14]'s equilibrium operator requires unconventional techniques of dynamic programming because the value function appears in the feasible set defined by constraints (2.1)-(2.2).
} 


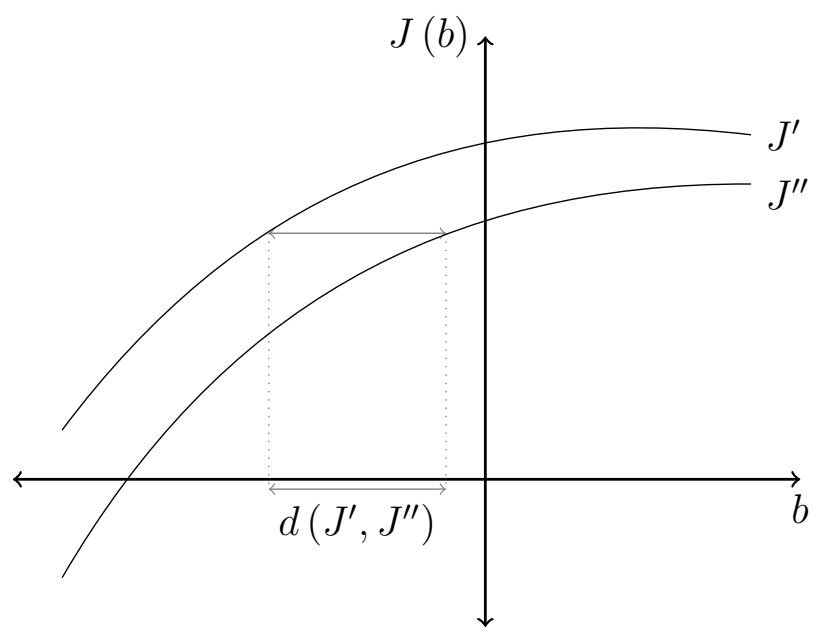

Figure 1: Horizontal distance

with the convention that $d_{f}\left(J^{\prime}, J^{\prime \prime}\right)=\infty$ when these constraints cannot be satisfied. As illustrated by Figure 1, this extended metric basically measures the horizontal distance between different candidate value functions. This approach mimics Aguiar and Amador [3]'s choice of working with the dual program, that is, with inverse functions. ${ }^{5}$

Claim 3 (Extended metric). Metric $d_{f}: \mathcal{J} \times \mathcal{J} \rightarrow \mathbb{R}^{*}$ satisfies the required properties:

(a) $d_{f}\left(J^{\prime}, J^{\prime \prime}\right)=0$ if and only if $J^{\prime}=J^{\prime \prime}$;

(b) $d_{f}\left(J^{\prime}, J^{\prime \prime}\right)=d_{f}\left(J^{\prime \prime}, J^{\prime}\right)$;

(c) $d_{f}\left(J^{\prime}, J^{\prime \prime}\right) \leq d_{f}\left(J^{\prime}, J\right)+d_{f}\left(J, J^{\prime \prime}\right)$.

${ }^{5}$ The minimum expenditure function is indeed the inverse of the indirect utility function. Measuring the horizontal distance between indirect utility functions corresponds to measuring the vertical distance between minimum expenditure functions. Notice that the minimum expenditure function is always unbounded, irrespectively of default, so raising an independent complication for the application of dynamic programming methods. 
Furthermore, for any $J$ in the order interval $\left[J^{\prime}, J^{\prime \prime}\right] \subset \mathcal{J}$,

$$
\max \left\{d_{f}\left(J^{\prime}, J\right), d_{f}\left(J, J^{\prime \prime}\right)\right\} \leq d_{f}\left(J^{\prime}, J^{\prime \prime}\right) .
$$

Proof. The proof of properties (a)-(c) is left to the reader. Let $\epsilon=d_{f}\left(J^{\prime}, J^{\prime \prime}\right)$, that is,

$$
J^{\prime}(s, b+\epsilon f(s)) \geq J^{\prime \prime}(s, b) \geq J^{\prime}(s, b-\epsilon f(s))
$$

and

$$
J^{\prime \prime}(s, b+\epsilon f(s)) \geq J^{\prime}(s, b) \geq J^{\prime \prime}(s, b-\epsilon f(s)) .
$$

Observing that $J^{\prime \prime} \geq J \geq J^{\prime}$, we obtain

$$
J(s, b+\epsilon f(s)) \geq J^{\prime}(s, b+\epsilon f(s)) \geq J^{\prime \prime}(s, b) \geq J(s, b-\epsilon f(s)) ;
$$

and, similarly,

$$
J(s, b+\epsilon f(s)) \geq J^{\prime}(s, b) \geq J^{\prime \prime}(s, b-\epsilon f(s)) \geq J(s, b-\epsilon f(s)) .
$$

This proves our claim.

\section{Contraction}

The operator is a contraction under the adapted metric when long-term interest rates exceed endowment growth. This condition is expressed in terms of the dominant root of the valuation operator. We briefly describe the approach, and refer to our previous paper [11] for a more comprehensive treatment. The advantage of our method is that it allows us to easily encompass time-varying interest rates by only considering a constant dominant root, as if the interest rate were effectively constant.

The valuation operator $\Pi: \mathbb{R}^{S} \rightarrow \mathbb{R}^{S}$ is defined as

$$
\Pi(f)(s)=\min _{b^{\prime} \in \mathbb{R}} Q^{*}\left(s, b^{\prime}\right)
$$


subject to

$$
f\left(s^{\prime}\right) \leq b^{\prime} \text { if } \mu\left(s, s^{\prime}\right)>0,
$$

where the default-free bond price is given by condition (3.1). The valuation operator computes the cost of fulfilling future obligations by means of the only available bond. Under a constant interest rate, it reduces to

$$
\Pi(f)(s)=\left(\frac{1}{1+r}\right) \max _{s^{\prime} \in S} f\left(s^{\prime}\right) \text { subject to } \mu\left(s, s^{\prime}\right)>0 \text {. }
$$

As in Perron-Frobenius theory, or in the spectral radius theory, the dominant root evaluates the action of the valuation operator relative to the endowment. Our Assumption 5 clarifies the precise meaning of long-term interest rates exceeding growth. It should be remarked that these conditions can be rarely expressed as a simple comparison between the average interest rate and the average growth rate under uncertainty (see Bloise et al. [11] for additional insights on this point). ${ }^{6}$

Assumption 5 (High interest rates). There exists $\rho$ in $(0,1)$ such that, for some strictly positive $f$ in $\mathbb{R}^{S}$ such that $\lambda_{u} y \geq f \geq \lambda_{l} y$ with $\lambda_{u}$ and $\lambda_{l}$ in $\mathbb{R}^{++}$,

$$
\Pi(f) \leq \rho f
$$

In the traditional framework with a constant interest rate, and no endowment growth,

$$
\rho=\frac{1}{1+r}
$$

Adjusting for a constant rate of growth $g$ in $\mathbb{R}^{+}$, the dominant root becomes

$$
\rho=\frac{1+g}{1+r} .
$$

\footnotetext{
${ }^{6} \mathrm{~A}$ comparison in terms of averages is unsatisfactory for the obvious reason that, as stated by Hansen and Scheinkman [15] while explaining their operator approach to long-run risk, 'long-term interest rates reflect a compounding of short-term rates'.
} 
With a time-varying interest rate governed by strictly positive transitions on a finite state space $S$,

$$
\rho=\max _{s \in S} \frac{1}{1+r(s)} .
$$

These are all frameworks in which a dominant root theory is, as a matter of fact, unnecessary because the interest rate exceeds growth period by period. However, in general, a more sophisticated approach is indispensable with time-varying interest rates and growth. The interest rate might exceed growth in some states, while falling below growth in other phases. The dominant root serves to weight these conflicting forces in the long term. In Appendix B and Appendix C we provide computational tools and further explain that the dominant root discerns ambiguous situations.

As in Aguiar and Amador [3], we consider a suitable upper bound on the debt issued by the sovereign corresponding to the greatest valuation of the sovereign's endowment, that is, its present value conditional on the most optimistic resolution of future uncertainty. Our Assumption 5 (High interest rates) ensures that this greatest valuation is in fact finite, whereas it will necessarily be infinite when it fails.

Assumption 6 (Debt limits). The upper bound on debt fulfils $N \leq \lambda \bar{N}$ for some $\lambda$ in $\mathbb{R}^{++}$, where $\bar{N}$ in $\mathbb{R}^{S}$ is the greatest valuation of the sovereign's endowment, that is, the minimal positive process satisfying

$$
\bar{N}(s)=y(s)+\Pi(\bar{N})(s) .
$$

We preliminarily observe that the identified bounds for indirect utility are within a finite distance. This is true because the upfront present value of future income more than compensates for the absence of borrowing privileges. Therefore, the extended metric is finitely valued on the relevant domain and this allows us to develop a contraction mapping approach. 
Claim 4 (Suitable interval). Under high interest rates (Assumption 5),

$$
d_{f}(\underline{J}, \bar{J}) \text { is finite. }
$$

Proof. We first show that, subject to Assumption 6, debt limit $N$ in $\mathbb{R}^{S}$ is bounded by (some expansion of) the strictly positive process $f$ in $\mathbb{R}^{S}$ given in Assumption 5. Setting $\bar{N}^{0}=0$ in $\mathbb{R}^{S}$, we recursively generate an increasing sequence by means of

$$
\bar{N}^{n}(s) \leq \bar{N}^{n+1}(s)=y(s)+\Pi\left(\bar{N}^{n}\right)(s) .
$$

Exploiting Assumption 5, we obtain

$$
\bar{N}^{n+1} \leq \frac{1}{\lambda_{l}}\left(\frac{1-\rho^{n+1}}{1-\rho}\right) f .
$$

Indeed, the inequality is trivially true for $n=0$. Assuming that it is satisfied for an arbitrary $n$ in $\mathbb{N}$, we obtain

$$
\begin{aligned}
\bar{N}^{n+1}(s) & \leq y(s)+\frac{1}{\lambda_{l}}\left(\frac{1-\rho^{n}}{1-\rho}\right) \Pi(f)(s) \\
& \leq \frac{1}{\lambda_{l}} f(s)+\frac{1}{\lambda_{l}}\left(\frac{1-\rho^{n}}{1-\rho}\right) \rho f(s) .
\end{aligned}
$$

Furthermore, the increasing sequence converges to the upper bound $\bar{N}$ in $\mathbb{R}^{S}$ which, by construction, satisfies the recursive property in Assumption 6. Thus, a bound exists and, at no loss of generality, we can assume that $N \leq f$.

For every state $s$ in $S$, there exists $\Delta b^{\prime}$ in $\mathbb{R}^{+}$such that

$$
Q^{*}\left(s, \Delta b^{\prime}\right)=f(s)
$$

and

$$
f\left(s^{\prime}\right) \leq \Delta b^{\prime} \text { if } \mu\left(s, s^{\prime}\right)>0 .
$$

We now show that any borrowing plan bounded by the debt limit $N$ in $\mathbb{R}^{S}$ can 
be replicated without borrowing given initial additional resources $f$ in $\mathbb{R}^{S}$. More precisely, we prove that

$$
\underline{J}(s, b) \geq \bar{J}(s, b-f(s)) .
$$

Here is the argument.

Let $T_{0}: \mathcal{J} \rightarrow \mathcal{J}$ be the Bellman operator corresponding to the program subject to no borrowing, and set $J$ in $\mathcal{J}$ as $J(s, b)=\bar{J}(s, b-f(s))$. We show that $\left(T_{0} J\right) \geq J$. By conventional arguments of dynamic programming, we then obtain that

$$
\underline{J}(s, b)=\lim _{n \in \mathbb{N}}\left(T_{0}^{n} J\right)(s, b) \geq J(s, b)=\bar{J}(s, b-f(s)),
$$

so establishing condition (*). To our purposes, we only need to consider any $b$ in $\mathbb{R}$ such that $\bar{J}(s, b-f(s))$ is finite. This value is attained by a plan such that

$$
Q^{*}\left(s, b^{\prime}\right)+c \leq y(s)+b-f(s)
$$

and

$$
-N\left(s^{\prime}\right) \leq b^{\prime} \text { if } \mu\left(s, s^{\prime}\right)>0,
$$

where the default-free pricing is given by (3.1). Shifting the bond position, we obtain

$$
Q^{*}\left(s, b^{\prime}+\Delta b^{\prime}\right)+c \leq y(s)+b,
$$

with $b^{\prime}+\Delta b^{\prime} \geq 0$. Furthermore,

$$
J\left(s^{\prime}, b^{\prime}+\Delta b^{\prime}\right)=\bar{J}\left(s^{\prime}, b^{\prime}+\Delta b^{\prime}-f\left(s^{\prime}\right)\right) \geq \bar{J}\left(s^{\prime}, b^{\prime}\right) \text { if } \mu\left(s, s^{\prime}\right)>0 .
$$

This is sufficient to establish that $\left(T_{0} J\right)(s, b) \geq J(s, b)$, as required to conclude our proof.

We next establish that the operator is indeed a contraction with Lipschitz con- 
stant $\rho$ in $(0,1)$, so extending Aguiar and Amador [3] to more general frameworks. This property is the reflection of the replication strategy originally used by Bulow and Rogoff [13] and recently exploited by Auclert and Rognlie [8] and Bloise et al. [10] to establish uniqueness of equilibrium. The sovereign can always replicate an optimal plan with large debt by reducing debt exposure and investing saved repayments in a fund yielding a constant return over time. This ensures that the distance in the introduced metric shrinks less than proportionally relative to the debt contraction.

To verify how the contraction property in the adapted metric is related to a replication policy, consider the budget constraint under value function $J^{\prime}$ in $\mathcal{J}$, that is,

$$
Q\left(s, b^{\prime} \mid J^{\prime}\right)+c \leq y(s)+b .
$$

Letting $\epsilon=d_{f}\left(J^{\prime}, J^{\prime \prime}\right)$, by the definition of the metric, we have

$$
J^{\prime}\left(s^{\prime}, b^{\prime}\right) \leq J^{\prime \prime}\left(s^{\prime}, b^{\prime}+\epsilon f\left(s^{\prime}\right)\right) \text { if } \mu\left(s, s^{\prime}\right)>0 .
$$

So, to afford the same utility level under the alternative value function $J^{\prime \prime}$ in $\mathcal{J}$, the sovereign would need additional resources $\epsilon f\left(s^{\prime}\right)$ in $\mathbb{R}$ at each future state $s^{\prime}$ in $S$ such that $\mu\left(s, s^{\prime}\right)>0$. Assumption 5 (High interest rates) ensures that the current cost of these additional resources is only $\rho \in f(s)$ in $\mathbb{R}$. Hence, were these additional resources available, any budget feasible plan under $J^{\prime}$ in $\mathcal{J}$ could be replicated under $J^{\prime \prime}$ in $\mathcal{J}$, which yields

$$
\left(T J^{\prime}\right)(s, b) \leq\left(T J^{\prime \prime}\right)(s, b+\rho \epsilon f(s))
$$

This establishes that $d_{f}\left(\left(T J^{\prime}\right),\left(T J^{\prime \prime}\right)\right) \leq \rho \epsilon$, as required for the contraction property.

Claim 5 (Contraction). For every $J^{\prime}$ and $J^{\prime \prime}$ in $\mathcal{J}$,

$$
d_{f}\left(\left(T J^{\prime}\right),\left(T J^{\prime \prime}\right)\right) \leq \rho d_{f}\left(J^{\prime}, J^{\prime \prime}\right)
$$


Proof. Letting $\epsilon=d_{f}\left(J^{\prime}, J^{\prime \prime}\right)$, we first show that $\left(T J^{\prime}\right)(s, b+\rho \epsilon f(s)) \geq\left(T J^{\prime \prime}\right)(s, b)$. Consider the plan achieving the maximum value $\left(T J^{\prime \prime}\right)(s, b)$ that satisfies the budget constraint

$$
Q\left(s, b^{\prime} \mid J^{\prime \prime}\right)+c \leq y(s)+b .
$$

By Assumption 5, there exists a shift satisfying

$$
Q\left(s, b^{\prime} \mid J^{\prime \prime}\right)+Q^{*}\left(s, \Delta b^{\prime}\right)+c \leq y(s)+b+\rho \epsilon f(s),
$$

where, by the definition of dominant root,

$$
\epsilon f\left(s^{\prime}\right) \leq \Delta b^{\prime} \text { if } \mu\left(s, s^{\prime}\right)>0 \text {. }
$$

From the latter, we obtain

$$
J^{\prime}\left(s^{\prime}, b^{\prime}+\Delta b^{\prime}\right) \geq J^{\prime}\left(s^{\prime}, b^{\prime}+\epsilon f(s)\right) \geq J^{\prime \prime}\left(s^{\prime}, b^{\prime}\right) \text { if } \mu\left(s, s^{\prime}\right)>0
$$

so that

$$
Q\left(s, b^{\prime}+\Delta b^{\prime} \mid J^{\prime}\right) \leq Q\left(s, b^{\prime} \mid J^{\prime \prime}\right)+Q^{*}\left(s, \Delta b^{\prime}\right)
$$

This establishes the inequality. We can argue similarly to verify that

$$
\left(T J^{\prime \prime}\right)(s, b) \geq\left(T J^{\prime}\right)(s, b-\rho \epsilon f(s))
$$

so concluding the proof.

We can now conclude the argument establishing existence of a unique fixed point. A conventional contraction mapping theorem cannot be applied, because completeness of space $\mathcal{J}$ in the extended metric is not ensured. ${ }^{7}$ However, mono-

\footnotetext{
${ }^{7}$ To obtain a complete space in the conventional supremum norm, Aguiar and Amador [3] assume that consumption remains in a compact interval. This is a rather restrictive property when growth is allowed. We instead impose no upper bound on consumption, and cannot rely on traditional arguments for the completeness of the underlying space.
} 
tonicity of the operator provides a supplementary property ensuring convergence to a fixed point, as in the traditional contraction mapping theorem.

Claim 6 (Fixed point). Operator $T: \mathcal{J} \rightarrow \mathcal{J}$ admits exactly one fixed point $J^{*}$ in the interval $[\underline{J}, \bar{J}] \subset \mathcal{J}$. Furthermore, the only fixed point $J^{*}$ belongs to the restricted domain $\mathcal{J}^{u}$. Finally, for every $J$ in $[\underline{J}, J] \subset \mathcal{J}$,

$$
\lim _{n \rightarrow \infty} d_{f}\left(\left(T^{n} J\right), J^{*}\right)=0
$$

Proof. Let $\underline{J}^{n}=\left(T^{n} \underline{J}\right)$ and $\bar{J}^{n}=\left(T^{n} \bar{J}\right)$, both elements of the restricted domain $\mathcal{J}^{u}$. By monotonicity of operator $T: \mathcal{J} \rightarrow \mathcal{J}$, the lower orbit is weakly increasing and the upper orbit is weakly decreasing with $\underline{J}^{n} \leq \bar{J}^{n}$. Furthermore, by the contraction property,

$$
d_{f}\left(\underline{J}^{n}, \bar{J}^{n}\right) \leq \rho^{n} d_{f}(\underline{J}, \bar{J})
$$

Finally, the lower orbit converges pointwise to $\underline{J}^{*}$ in $\mathcal{J}$, and the upper orbit converges pointwise to $\bar{J}^{*}$ in $\mathcal{J}^{u}$, that is, upper semicontinuity is preserved in the limit (see Aliprantis and Border [6, Lemma 2.41]). By the additional property of the extended metric in Claim 3, we obtain

$$
\rho^{-1} d_{f}\left(\left(T \bar{J}^{*}\right), \bar{J}^{n+1}\right) \leq d_{f}\left(\bar{J}^{*}, \bar{J}^{n}\right) \leq d_{f}\left(\underline{J}^{n}, \bar{J}^{n}\right) \leq \rho^{n} d_{f}(\underline{J}, \bar{J})
$$

which suffices to prove the existence of a fixed point by the triangle inequality,

$$
d_{f}\left(\left(T \bar{J}^{*}\right), \bar{J}^{*}\right) \leq d_{f}\left(\left(T \bar{J}^{*}\right), \bar{J}^{n}\right)+d_{f}\left(\bar{J}^{*}, \bar{J}^{n}\right) .
$$

We so establish that $\bar{J}^{*}$ in $\mathcal{J}^{u}$ is a fixed point and, by the contraction property, the fixed point is unique in $[\underline{J}, \bar{J}] \subset \mathcal{J}$. Finally, for every arbitrary $J$ in $[\underline{J}, \bar{J}] \subset \mathcal{J}$,

$$
d_{f}\left(\left(T^{n} J\right), J^{*}\right) \leq \rho^{n} d_{f}\left(J, J^{*}\right) \leq \rho^{n} d_{f}(\underline{J}, \bar{J})
$$

so proving our claim. Notice that, in particular, this implies that $d_{f}\left(\underline{J}^{*}, \bar{J}^{*}\right)=0$, so that the two limits coincide. 


\section{Conclusion}

We have established existence and uniqueness of equilibrium in a general version of Eaton and Gersovitz [14]'s sovereign debt model with time-varying interest rates and growth. The method exploited a contraction property of the canonical equilibrium operator in an adapted metric, mirroring primitive arbitrage opportunities. The contraction property relies on long-term interest rates exceeding economic growth, and it might fail otherwise. This fact sheds light on the occurrence of sovereign debt crises (multiple equilibria) under low interest rates, an issue studied by Bloise and Vailakis [12].

\section{Appendix A. Robustness}

High implied interest rates (Assumption 5) are neither necessary for a unique equilibrium nor for the contraction property. They are only needed to ensure the existence of a pure arbitrage: any plan with high borrowing can be replicated with low borrowing given a corresponding contraction of initial debt. When interest rates are not high, this pure arbitrage disappears. However, by continuity, in an appropriate neighborhood, gains from a reduced initial debt still exceed the losses due to a diminished borrowing ability, and some sort of contraction property persists. We briefly explore this issue of robustness.

For tractability we assume a finite state space $S$ and we only perturb transition probabilities explicitly. Perturbations of the pricing kernel and other fundamentals could be accommodated with minor changes. We thus consider a sequence of Markov transitions $\mu_{n}: S \rightarrow \Delta(S)$ such that

$$
\lim _{n \rightarrow \infty} \mu_{n}\left(s, s^{\prime}\right)=\mu\left(s, s^{\prime}\right)
$$

Notice that, although the stochastic discount factor is fixed, interest rates might not be high along the sequence, even when they are certainly high in the limit. As illustrated in the next example, this entails no paradox: high interest rates depend on the ergodic set, which might dramatically shrink in the limit. 
Example 1 (Negative interest on small probability events). Consider the Markov space $S=\{\alpha, \beta\}$ with

$$
r(\alpha)>0>r(\beta)
$$

The sequence of Markov transitions is given by

$$
\mu_{n}(\alpha, \beta)=\mu_{n}(\beta, \beta)=\frac{1}{n} \text { and } \mu_{n}(\alpha, \alpha)=\mu_{n}(\beta, \alpha)=\frac{n-1}{n} .
$$

By direct computation,

$$
\rho_{n}=\frac{1}{1+r(\beta)}>1>\frac{1}{1+r(\alpha)}=\rho,
$$

where $\rho_{n}$ and $\rho$ in $\mathbb{R}^{++}$are the dominant roots appearing in Assumption 5, respectively, along the sequence and in the limit. Hence, interest rates are low along the sequence, and high in the limit.

To ascertain robustness, we assume that the limit Markov transition $\mu: S \rightarrow$ $\Delta(S)$ satisfies high interest rates (Assumption 5). We also confine attention to a constant upper bound on debt, that is, $N(s)=N$ for all $s$ in $S$. We then establish, subject to a Strong Inada Condition, that all equilibria converge to the unique limit equilibrium. This does not ensure uniqueness in the perturbed economy. However, any residual multiplicity bears a negligible effect on the sovereign's ability to borrow.

Assumption 7 (Strong Inada Condition). For every $s$ in $S$,

$$
(1-\delta) u(0)+\delta \sup _{c \in \mathbb{R}^{+}} u(c)<J^{D}(s)<\sup _{c \in \mathbb{R}^{+}} u(c)
$$

Claim 7 (Almost uniqueness). Under the maintained assumptions,

$$
\limsup _{n \rightarrow \infty} \max _{s \in S}\left|g_{n}^{\prime}(s)-g_{n}^{\prime \prime}(s)\right|=0
$$

where $g_{n}^{\prime}$ and $g_{n}^{\prime \prime}$ in $\mathbb{R}^{S}$ are equilibrium default thresholds in the perturbed econ- 
omy such that

$$
J_{n}^{\prime}\left(s,-g_{n}^{\prime}(s)\right)=J_{n}^{\prime \prime}\left(s,-g_{n}^{\prime \prime}(s)\right)=J^{D}(s)
$$

Proof. As equilibrium is unique in the limit, it suffices to show that any sequence of equilibrium default thresholds $g_{n}$ in $\mathbb{R}^{S}$ converges, possibly extracting a subsequence, to equilibrium default thresholds $g$ in $\mathbb{R}^{S}$. As this is more convenient (see Auclert and Rognlie [8, Appendix A]), we work with the sequential program subject to given default thresholds. At equilibrium, indeed, by monotonicity of the indirect utility,

$$
J(s, b) \geq J^{D}(s) \text { if and only if } b \geq-g(s) .
$$

This ensures the basic equivalence.

Let $\mathcal{S}(s)$ be the space of histories $s^{t}=\left(s_{0}, \ldots, s_{t}\right)$ given an initial state $s_{0}=s$ in $S$. Along a history $s^{t}$ in $\mathcal{S}(s)$ with no default, the budget constraint is

$$
Q\left(s_{t}, b\left(s^{t}\right) \mid g\right)+c\left(s^{t}\right) \leq y\left(s_{t}\right)+b\left(s^{t-1}\right) .
$$

The risk-premium on sovereign bonds is determined according to

$$
Q\left(s, b^{\prime} \mid g\right)=\left(\frac{1}{1+r(s)}\right) b^{\prime}-\sum_{s^{\prime} \in D\left(s, b^{\prime} \mid g\right)} \mu\left(s, s^{\prime}\right) m\left(s, s^{\prime}\right) \min \left\{b^{\prime}, 0\right\}
$$

where the default event is

$$
D\left(s, b^{\prime} \mid g\right)=\left\{s^{\prime} \in S: b^{\prime}<-g\left(s^{\prime}\right)\right\}
$$

Whenever default occurs, the reservation value is $J^{D}\left(s_{t}\right)$ in $\mathbb{R}$. The sovereign maximizes utility subject to this sequence of budget constraints, and to the constant upper bound on debt, given initial wealth $b$ in $\mathbb{R}$. This gives rise to an indirect utility value $J(s, b \mid g)$ in $\mathbb{R}^{*}$. 
By upper hemicontinuity of the budget set, we obtain

$$
J(s,-g(s) \mid g) \geq \lim _{n \rightarrow \infty} J_{n}\left(s,-g_{n}(s) \mid g\right)=\lim _{n \rightarrow \infty} J_{n}\left(s,-g_{n}(s)\right)=J^{D}(s) .
$$

Suppose that $J(s,-g(s) \mid g)>J^{D}(s)$ and notice that, by the Strong Inada Condition, consumption is certainly strictly positive as long as default has not occurred. The optimal plan in the limit, conditional on no default, satisfies

$$
Q\left(s_{t}, b\left(s^{t}\right) \mid g\right)+c\left(s^{t}\right)=y\left(s_{t}\right)+b\left(s^{t-1}\right) .
$$

For all sufficiently large $n$ in $\mathbb{N}$, we approximate this optimal plan by setting

$$
Q_{n}\left(s_{t}, b_{n}\left(s^{t}\right) \mid g_{n}\right)+c_{n}\left(s^{t}\right)=y\left(s_{t}\right)+b_{n}\left(s^{t-1}\right)
$$

where

$$
b_{n}\left(s^{t}\right)=b\left(s^{t}\right)+\max _{s \in S}\left(g(s)-g_{n}(s)\right)^{+} .
$$

We can assume that default occurs if the approximate consumption is negative, as it might only happen in the remote future for large $n$ in $\mathbb{N}$. By discounting, and continuity of the utility, we only have to prove that the perturbed consumption is close to the limit consumption over a sufficiently large finite horizon. For this purpose, it suffices to show that

$$
\lim _{n \rightarrow \infty} Q_{n}\left(s_{t}, b_{n}\left(s^{t}\right) \mid g_{n}\right)=Q\left(s_{t}, b\left(s^{t}\right) \mid g\right) .
$$

This would imply that, for any large $n$ in $\mathbb{N}, J_{n}\left(s,-g_{n}(s) \mid g\right)>J^{D}(s)$, thus yielding a contradiction. We so verify continuity of the pricing.

Fix a history $s^{t}$ in $\mathcal{S}(s)$ and notice that

$$
b\left(s^{t}\right)<-g\left(s_{t+1}\right) \text { if } b_{n}\left(s^{t}\right)<-g_{n}\left(s_{t+1}\right) .
$$


This implies that, for every sufficiently large $n$ in $\mathbb{N}$,

$$
D\left(s_{t}, b_{n}\left(s_{t}\right) \mid g_{n}\right)=D\left(s_{t}, b\left(s_{t}\right) \mid g\right) .
$$

As transition probabilities converge, this concludes our proof.

\section{Appendix B. Spectral radius}

We complete our analysis by illustrating a method to ascertain whether the hypothesis of implied interest rates is satisfied. This approach is suitable for empirical applications and relies on the approximation of the spectral radius of the valuation operator, interpretable as the yield-to-maturity of a long-term discount bond indexed to growth. To apply this technique, we preliminarily need to further restrict the valuation operator by imposing bounds on the time-variation of the interest rate relative to growth. This further assumption is immediately satisfied when state space $S$ is finite. ${ }^{8}$

Assumption 8 (Bounds on interest rates). There exist $\lambda_{l}$ and $\lambda_{h}$ in $\mathbb{R}^{++}$such that

$$
\lambda_{l} y \leq \Pi(y) \leq \lambda_{h} y
$$

We consider the space of all processes that are bounded relative to the endowment, that is,

$$
L(y)=\left\{v \in \mathbb{R}^{S}:|v| \leq \lambda y \text { for some } \lambda \in \mathbb{R}^{+}\right\}
$$

This space is endowed with the canonical supremum norm given by

$$
\|v\|=\inf \left\{\lambda \in \mathbb{R}^{+}:|v| \leq \lambda y\right\} .
$$

\footnotetext{
${ }^{8} \mathrm{~A}$ mathematical treatment of the spectral radius of positive linear operators can be found in Aliprantis and Border [6, Chapter 20]. For our purposes, we need an extension of this theory to positive sublinear operators.
} 
The spectral radius of the (restricted) valuation operator $\Pi: L(y) \rightarrow L(y)$ is

$$
r(\Pi)=\lim _{n \in \mathbb{N}} \sqrt[n]{\left\|\Pi^{n}\right\|}
$$

where the operator norm is defined as

$$
\|\Pi\|=\sup _{v \in L(y)}\{\|\Pi(v)\|:\|v\| \leq 1\}
$$

We translate the hypothesis of high interest rates in terms of spectral radius. To this purpose, we need to exploit a well-known property of the supremum norm of monotone sublinear operators, of which we provide a simple proof for completeness. We then establish that the condition that interest rate exceeds growth in the long term can be equivalently expressed as $r(\Pi)<1$.

Claim 8 (Operator norm). For every $n$ in $\mathbb{N}$,

$$
\left\|\Pi^{n}\right\|=\left\|\Pi^{n}(y)\right\|
$$

Proof. The operator norm is given by

$$
\left\|\Pi^{n}\right\|=\sup _{v \in[-y, y]}\left\|\Pi^{n}(v)\right\|
$$

Notice that, by monotone sublinearity,

$$
\left|\Pi^{n}(v)\right| \leq \Pi^{n}(|v|)
$$

Indeed,

$$
-\Pi^{n}(|v|) \leq \Pi^{n}(-|v|) \leq \Pi^{n}(v) \leq \Pi^{n}(|v|)
$$

It follows that

$$
\left\|\Pi^{n}(y)\right\| \leq\left\|\Pi^{n}\right\| \leq \sup _{v \in[-y, y]}\left\|\Pi^{n}(|v|)\right\|=\left\|\Pi^{n}(y)\right\|,
$$


so proving the claim.

Claim 9 (Spectral radius). Assumption 5 (High interest rates) is satisfied if and only if $r(\Pi)<1$.

Proof of necessity. When Assumption 5 is satisfied, by iterating, we obtain

$$
\rho^{n} f \geq \Pi^{n}(f)
$$

Using the bounds provided in Assumption 5, and exploiting monotonicity, this yields

$$
\rho^{n}\left(\frac{\lambda_{h}}{\lambda_{l}}\right) y \geq \Pi^{n}(y)
$$

which in turn implies

$$
\rho^{n}\left(\frac{\lambda_{h}}{\lambda_{l}}\right) \geq\left\|\Pi^{n}\right\| .
$$

Taking the root,

$$
\rho \sqrt[n]{\frac{\lambda_{h}}{\lambda_{l}}} \geq \sqrt[n]{\left\|\Pi^{n}\right\|}
$$

thus proving that $\rho \geq r(\Pi)$.

Proof of sufficiency. Pick any $r$ in $(r(\Pi), 1)$. For any sufficiently large $n$ in $\mathbb{N}$,

$$
\Pi^{n}(y) \leq\left\|\Pi^{n}\right\| y \leq r^{n} y
$$

Defining

$$
f^{n}=y+\Pi(y)+\cdots+\Pi^{n}(y)
$$

notice that, by sublinearity,

$$
y+\Pi\left(f^{n}\right) \leq f^{n+1}
$$

The process monotonically (order) converges to $f$ in $L(y)$ because condition (**) 
provides a geometric upper bound eventually. We so obtain that, in the limit,

$$
y+\Pi(f) \leq f
$$

Furthermore, for some sufficiently large $\rho$ in $(0,1)$,

$$
(1-\rho) f \leq y
$$

We so conclude that

$$
(1-\rho) f+\Pi(f) \leq f \text { if and only if } \Pi(f) \leq \rho f .
$$

This proves our claim.

\section{Appendix C. Compound interest rate}

Consider an irreducible Markov transition $\mu: S \rightarrow \Delta(S)$ on a finite state space $S$. As usual, we endow state space $S$ with the immediate successor relation:

$$
s^{\prime} \succ s \text { if and only if } \mu\left(s, s^{\prime}\right)>0 \text {. }
$$

A cycle $c$ in $\mathcal{C}$ is a finite sequence $\left(s_{1}, \ldots, s_{k}, \ldots, s_{n}\right)$ of distinct states in $S$ such that

$$
s_{1} \succ s_{n} \succ \cdots \succ s_{k} \succ \cdots \succ s_{1}
$$

As the state space $S$ is finite, so is the set of all irreducible cycles $\mathcal{C}$.

We provide an operational characterization of the dominant root condition necessary for the contraction. It is worth noticing that the interest rate might be negative for a long phase even though positive over the long term, that is, we can have $r(s)<0$ for several states in $S$ when Assumption 5 holds true. We complement our presentation with a simple example (Example 2).

Claim 10 (Discounting). Assumption 5 (High interest rates) is satisfied if and only 
if, for some $\rho$ in $(0,1)$,

$$
\max _{c \in \mathcal{C}} \sqrt[n]{\left(\frac{1}{1+r\left(s_{1}\right)}\right) \cdots\left(\frac{1}{1+r\left(s_{n}\right)}\right)}=\rho .
$$

Proof. By direct inspection, for every $f$ in $\mathbb{R}^{S}$, we have

$$
\Pi(f)(s)=\left(\frac{1}{1+r(s)}\right) \max _{s^{\prime} \succ s} f\left(s^{\prime}\right) .
$$

Thus, defining $f^{0}=1$ and $f^{n+1}=\Pi\left(f^{n}\right)$, we obtain

$$
f^{n+1}(s)=\left(\frac{1}{1+r(s)}\right) \max _{s^{\prime} \succ s} f^{n}\left(s^{\prime}\right) .
$$

By the spectral radius characterization (see Claim 9), we have to show that condition (***) holds true if and only if $\lim _{n \rightarrow \infty} \sqrt[n]{\left\|\Pi^{n}(\mathbf{1})\right\|}<1$ if and only if $\lim _{n \rightarrow \infty} \sqrt[n]{f^{n}(s)}<1$ for every $s$ in $S$.

Let $\mathcal{S}^{n}$ be the space of all finite paths $\left(s_{1}, \ldots, s_{k}, \ldots, s_{n}\right)$ of states in $S$ such that

$$
s_{n} \succ \cdots \succ s_{k} \succ \cdots \succ s_{1},
$$

and let $\mathcal{S}^{n}(s)$ be its restriction to a given initial state $s$ in $S$. We claim that

$$
f^{n}(s)=\max _{\sigma \in \mathcal{S}^{n}(s)}\left[\left(\frac{1}{1+r\left(s_{1}\right)}\right) \cdots\left(\frac{1}{1+r\left(s_{n}\right)}\right)\right] .
$$

Indeed, it is clear that, as $f^{0}=1$,

$$
f^{1}(s)=\left(\frac{1}{1+r(s)}\right) \max _{s^{\prime} \succ s} f^{0}\left(s^{\prime}\right)=\max _{\sigma \in \mathcal{S}^{1}(s)}\left(\frac{1}{1+r(s)}\right) .
$$


Furthermore, for any $n$ in $\mathbb{N}$,

$$
\begin{aligned}
f^{n+1}(s) & =\max _{\sigma \in \mathcal{S}^{n+1}(s)}\left[\left(\frac{1}{1+r\left(s_{1}\right)}\right) \cdots\left(\frac{1}{1+r\left(s_{n+1}\right)}\right)\right] \\
& =\left(\frac{1}{1+r(s)}\right) \max _{s^{\prime} \succ s} \max _{\sigma^{\prime} \in \mathcal{S}^{n}\left(s^{\prime}\right)}\left[\left(\frac{1}{1+r\left(s_{1}^{\prime}\right)}\right) \cdots\left(\frac{1}{1+r\left(s_{n}^{\prime}\right)}\right)\right] \\
& =\left(\frac{1}{1+r(s)}\right) \max _{s^{\prime} \succ s} f^{n}\left(s^{\prime}\right)
\end{aligned}
$$

confirming our conjecture. We so obtain that, for every $n$ in $\mathbb{N}$, there exists a path $\sigma$ in $\mathcal{S}^{n}(s)$ such that

$$
f^{n}(s)=\left[\left(\frac{1}{1+r\left(s_{1}\right)}\right) \cdots\left(\frac{1}{1+r\left(s_{n}\right)}\right)\right] .
$$

We show that, for every sufficiently large $n$ in $\mathbb{N}$,

$$
f^{n}(s) \leq \rho^{n-\operatorname{card}(S)}\left[\max _{s^{\prime} \in S}\left(\frac{1}{1+r\left(s^{\prime}\right)}\right)\right]^{\operatorname{card}(S)} .
$$

When $n>$ card $(S)$, some state in $S$ necessarily repeats itself along a finite path $\sigma$ in $\mathcal{S}^{n}(s)$, that is,

$$
\sigma=\left(s_{1}, \ldots, s_{k+1}, \ldots, s_{k+m}, s_{k+m+1}, \ldots, s_{n}\right), \text { with } s_{k+1}=s_{k+m+1} .
$$

We can assume that $\left(s_{k+1}, \ldots, s_{k+m}\right)$ is an irreducible cycle in $\mathcal{C}$, because if not some state repeats itself in this subpath and we can reiterate the argument. Removing the irreducible cycle, we conclude that

$$
\left(s_{1}, \ldots, s_{k}, s_{k+m+1}, \ldots, s_{n}\right) \in \mathcal{S}^{n-m}(s)
$$

and

$$
f^{n}(s) \leq \rho^{m}\left[\max _{s^{\prime} \in S}\left(\frac{1}{1+r\left(s^{\prime}\right)}\right)\right]^{n-m} .
$$

We can so reproduce the argument as long as, after removing the last irreducible 
cycle, we are left with enough states so that $n-m>\operatorname{card}(S)$, because some state will repeat itself in the residual finite path. By induction, this proves our claim.

Example 2 (Negative interest rate). We provide a simple example with two Markov states, $S=\{\alpha, \beta\}$. Transition probabilities are $\mu(\alpha, \alpha)=0, \mu(\alpha, \beta)=1$, $\mu(\beta, \alpha)>0$ and $\mu(\beta, \beta)>0$. Under the stated assumptions, the space of irreducible cycles is

$$
\mathcal{C}=\{\beta,(\alpha, \beta),(\beta, \alpha)\} .
$$

The discounting condition is satisfied if and only if

$$
\left(\frac{1}{1+r(\beta)}\right)<1 \text { and } \sqrt{\left(\frac{1}{1+r(\alpha)}\right)\left(\frac{1}{1+r(\beta)}\right)}<1 \text {. }
$$

These restrictions can be certainly fulfilled even if $r(\alpha)<0$.

\section{References}

[1] Abel, A. B., Mankiw, G. N., Summers, L. H., Zeckhauser, R. J., 1989. Assessing dynamic efficiency: Theory and evidence. Review of Economic Studies 56 (1), 1-19.

[2] Aguiar, M., Amador, M., 2014. Sovereign debt. In: Gopinath, G., Helpman, E., Rogoff, K. (Eds.), Handbook of International Economics. Vol. 4. Elsevier, Ch. 11, pp. 647-687.

[3] Aguiar, M., Amador, M., 2019. A contraction for sovereign debt models. Journal of Economic Theory 183, 842-875.

[4] Aguiar, M., Amador, M., Hopenhayn, H., Werning, I., 2019. Take the short route: Equilibrium default and debt maturity. Econometrica 87 (2), 423-462.

[5] Aguiar, M., Gopinath, G., 2006. Defaultable debt, interest rates, and the current account. Journal of International Economics 69 (1), 64-83. 
[6] Aliprantis, C. D., Border, K. C., 2006. Infinite Dimensional Analysis, 3rd Edition. Berlin: Springer.

[7] Arellano, C., 2008. Default risk and income fluctuations in emerging economies. American Economic Review 98 (3), 690-712.

[8] Auclert, A., Rognlie, M., 2016. Unique equilibrium in the Eaton-Gersovitz model of sovereign debt. Journal of Monetary Economics 84, 134-146.

[9] Blanchard, O. J., 2018. Public debt and low interest rates. American Economic Review 109 (4), 1197-1229.

[10] Bloise, G., Polemarchakis, H., Vailakis, Y., 2017. Sovereign debt and default incentives with uninsurable risks. Theoretical Economics 12 (3), 1121-1154.

[11] Bloise, G., Polemarchakis, H., Vailakis, Y., 2021. Sustainable debt, forthcoming at Theoretical Economics.

[12] Bloise, G., Vailakis, Y., November 2019. Sovereign debt crises and low interest rates, available at SSRN: http://dx.doi.org/10.2139/ssrn.3499620.

[13] Bulow, J., Rogoff, K., 1989. Sovereign debt: Is to forgive to forget? American Economic Review 79 (1), 43-50.

[14] Eaton, J., Gersovitz, M., 1981. Debt with potential repudiation: Theoretical and empirical analysis. Review of Economic Studies 48 (2), 289-309.

[15] Hansen, L. P., Scheinkman, J. A., 2009. Long-term risk: An operator approach. Econometrica 77 (1), 177-234.

[16] Jordà, O., Knoll, K., Kuvshinov, D., Schularick, M., Taylor, A. M., 2019. The rate of return on everything: 1870-2015. Quarterly Journal of Economics 134 (3), 1225-1298.

[17] Passadore, J., Xandri, J. P., 2020. Robust predictions in dynamic policy games, working paper. 